\title{
Complete Ionisation of the Neutral Gas in High Redshift Radio Galaxies and Quasars
}

\author{
S. J. Curran ${ }^{1,2}$ and M. T. Whiting ${ }^{3}$ \\ ${ }^{1}$ Sydney Institute for Astronomy, The University of Sydney, NSW 2006, Australia \\ ${ }^{2}$ ARC Centre of Excellence for All-sky Astrophysics (CAASTRO) \\ email: sjc@physics. usyd.edu.au \\ ${ }^{3}$ CSIRO Astronomy and Space Science, PO Box 76, Epping NSW 1710, Australia \\ email: Matthew. Whiting@csiro.au
}

\begin{abstract}
Cool neutral gas provides the raw material for all star formation in the Universe, and yet, from a survey of the hosts of high redshift radio galaxies and quasars, we find a complete dearth of atomic ( $\mathrm{H} \mathrm{I} 21-\mathrm{cm})$ and molecular $\left(\mathrm{OH}, \mathrm{CO}, \mathrm{HCO}^{+} \& \mathrm{HCN}\right)$ absorption at redshifts $z \gtrsim 3$ (Curran et al. 2008). Upon a thorough analysis of the optical photometry, we find that all of our targets have ionising $(\lambda \leqslant 912 \AA)$ ultra-violet continuum luminosities of $L_{\mathrm{UV}} \gtrsim 10^{23} \mathrm{~W}$ $\mathrm{Hz}^{-1}$. We therefore attribute this deficit to the traditional optical selection of targets biasing surveys towards the most ultra-violet luminous objects, where the intense radiation excites the neutral gas to the point where it cannot engage in star formation (Curran \& Whiting 2010). However, this hypothesis does not explain why there is a critical luminosity, rather than a continuum where the detections gradually become fewer and fewer as the harshness of the radiation increases. We show that by placing a quasar within a galaxy of gas there is always a finite ultra-violet luminosity above which all of the gas is ionised. This demonstrates that these galaxies are probably devoid of star-forming material rather than this being at abundances below the sensitivity limits of current radio telescopes.
\end{abstract}

Keywords. galaxies: active — galaxies: ISM — radio lines: galaxies — ultraviolet: galaxies

For a cloud of hydrogen containing an ionising source, the equilibrium between photoionsation and recombination of protons and electrons in a nebula is given by

$$
\int_{\nu_{\text {ion }}}^{\infty} \frac{L_{\nu}}{h \nu} d \nu=4 \pi \int_{0}^{r_{\text {ion }}} n_{\mathrm{p}} n_{\mathrm{e}} \alpha_{A} r^{2} d r=4 \pi \alpha_{\mathrm{A}} n_{0}^{2} \int_{0}^{r_{\text {ion }}} e^{-2 r / R} r^{2} d r,
$$

for a gas density $n_{\mathrm{p}}=n_{\mathrm{e}}=n=n_{0} e^{-r / R}$, where $n_{0}\left(=10 \mathrm{~cm}^{-3}\right)$ is the value at $r=0$ and $R$ is a scale-length describing the rate of decay of this with radius. For the observed critical value of $\int_{\nu_{\text {ion }}}^{\infty}\left(L_{\nu} / h \nu\right) d \nu=3 \times 10^{56}$ ionising photons $\sec ^{-1}\left(L_{\mathrm{UV}} \sim 10^{23} \mathrm{~W}\right.$ $\mathrm{Hz}^{-1}$ ), this gives a scale-length of $R=3 \mathrm{kpc}$, which is the value found for the $\mathrm{H}$ i in the Milky Way, which has an exponential profile with a similar $n_{0}=13.4 \mathrm{~cm}^{-3}$ (Kalberla \& Kerp 2009). That is, the observed critical luminosity is sufficient to ionise all of the gas in a large spiral galaxy, thus explaining why neutral gas is not detected in high redshift optically selected sources (Curran \& Whiting 2012). Therefore, even the SKA is unlikely to detect 21-cm absorption within the host galaxies of these objects.

\section{References}

Curran, S. J. \& Whiting, M. T. 2010, ApJ, 712, 303

Curran, S. J. \& Whiting, M. T. 2012, ApJ, in press (arXiv:1204.2881)

Curran, S. J., Whiting, M. T., Wiklind, T., Webb, J. K., Murphy, M. T., \& Purcell, C. R. 2008, MNRAS, 391, 765

Kalberla, P. M. W. \& Kerp, J. 2009, ARAA, 47, 27 